\title{
A Statistical Study of Temporally Smoothed Wavelet Coherence
}

\author{
E. A. K. Cohen and A. T. Walden, Member, IEEE
}

\begin{abstract}
The use of the wavelet coherence of two series in hypothesis testing relies on some sort of smoothing being carried out in order that the coherence estimator is not simply unity. A previous study considered averaging via the use of multiple Morse wavelets. Here we consider time-domain smoothing and use of a single Morlet wavelet. Since the Morlet wavelet is complex-valued, we derive analytic results for the case of wavelet coherence calculated from complex-valued, jointly stationary and Gaussian time series. The temporally smoothed wavelet coherence can be written in terms of Welch's overlapping segment averaging (WOSA) spectrum estimators, and by using multitaper equivalent representations for the WOSA estimators we show that Goodman's distribution is appropriate asymptotically, and readily derive the appropriate degrees of freedom. The theoretical results are verified via simulations and illustrated using solar physics data.
\end{abstract}

Index Terms-Coherence, Goodman's distribution, Morlet wavelet, temporal smoothing, wavelet coherence, WOSA.

\section{INTRODUCTION}

$\mathbf{T}$ IME series are often considered to derive from an ensemble of subprocesses, each operating at a characteristic scale (e.g., [18]) and wavelet analysis has a fundamental role "in decomposing structures at different scales hidden in time series data" [8]. The use of a wavelet coherence measure, WCOH say, in the scale-time (wavelet) domain, continues to be of great interest in many fields of science [2], [12], [13], [17], [22], [26]. The continuous wavelet transform (CWT) $W(a, b ; Z, \psi)$ at scale $a$, for $0<|a|<\infty$, and translation or time $b \in \mathbb{R}$ is defined as (e.g., [21])

$$
W(a, b ; Z, \psi)=\frac{1}{\sqrt{|a|}} \int_{-\infty}^{\infty} Z(t) \psi^{*}\left(\frac{t-b}{a}\right) \mathrm{d} t
$$

where $W(a, b ; Z, \psi)$ is the wavelet transform of a signal $Z(t)$, $\psi(\cdot)$ is the analyzing wavelet, and ${ }^{*}$ denotes complex conjugation. Given two jointly stationary signals $Z_{1}(t)$ and $Z_{2}(t)$, and with ' $\mathcal{S}$ ' representing a smoothing step, a wavelet coherence estimator at a particular scale $a$ and time $b$ in the time-scale domain can be expressed as (e.g., [22])

$$
\frac{\left|\mathcal{S}\left\{W\left(a, b ; Z_{1}, \psi\right) W^{*}\left(a, b ; Z_{2}, \psi\right)\right\}\right|^{2}}{\left[\mathcal{S}\left\{\left|W\left(a, b ; Z_{1}, \psi\right)\right|^{2}\right\}\right]\left[\mathcal{S}\left\{\left|W\left(a, b ; Z_{2}, \psi\right)\right|^{2}\right\}\right]} .
$$

Without smoothing this quantity is identically unity [15].

Manuscript received October 22, 2009; accepted January 27, 2010. Date of publication February 17, 2010; date of current version May 14, 2010. The associate editor coordinating the review of this manuscript and approving it for publication was Prof. Andreas Jakobsson. The work of E. A. K. Cohen was supported by the EPSRC (UK).

The authors are with the Department of Mathematics, Imperial College London, London SW7 2BZ, U.K. (e-mail: edward.cohen07@imperial.ac.uk; a.walden@imperial.ac.uk).

Digital Object Identifier 10.1109/TSP.2010.2043139
Used in combination with a Morlet wavelet $\psi$, smoothing has been carried out over time, $b$, followed by a smoothing over scale $a$ [2], [22], [26], or more simply using just a smoothing over time [12], [13], [17]. Under the null hypothesis that the two processes are jointly stationary and Gaussian with a given second-order structure, the full statistical analysis effects of such smoothing have not been determined in either case and statistical properties have only been determined by Monte Carlo simulation, e.g., [11], [13], and [22]. For example, [11] look for intermittent coherent oscillations against background red noise (low frequency) spectra. The background processes thus define the null hypothesis. If the computed $\mathrm{WCOH}$ value at $(a, b)$ exceeds the chosen simulated critical value, then the oscillation is declared present at that scale and time.

Recently, statistical results were derived for wavelet coherence when smoothing is achieved by averaging over samples generated by using multiple Morse wavelets [6]. In this paper, we examine the statistical properties of a $\mathrm{WCOH}$ estimator using a single Morlet wavelet and temporal smoothing over a small localized time interval centered at the translation of interest $b$. We call this the TWCOH estimator. With this approach the numerator of (2) is

$$
\begin{aligned}
& \mathcal{S}\left\{W\left(a, b ; Z_{1}, \psi\right) W^{*}\left(a, b ; Z_{2}, \psi\right)\right\} \\
& \quad=\frac{1}{\delta} \int_{b-\frac{\delta}{2}}^{b+\frac{\delta}{2}} W\left(a, \tau ; Z_{1}, \psi\right) W^{*}\left(a, \tau ; Z_{2}, \psi\right) \mathrm{d} \tau
\end{aligned}
$$

with obvious changes for the denominator. It was recognized in [13] that an expression like (3) could be interpreted as a continuous-time Welch's (or weighted) overlapping segment averaging (WOSA) estimator [5], [20]. We use this interpretation along with a discrete-time formulation as the first step in our approach. Then we cast the WOSA estimator into a multitaper-type formulation which allows us to derive highly accurate degrees of freedom estimates. In so doing we are also able to remove assumptions and approximations made by Bortel and Sovka [1] in their recent study of the statistical distribution of magnitude squared coherence estimated with segment overlapping. Since the Morlet wavelet is complex-valued, we derive analytic results for the case of wavelet coherence calculated from complex-valued, jointly stationary and Gaussian time series. The distribution for the TWCOH estimator, (2), is found to be that given by Goodman [9, eq. (5.54)], hereafter called Goodman's distribution.

The paper is arranged as follows. In Section II we describe the particular Morlet wavelet used and show how the numerator and denominator expressions in (2) can be written in a continuous-time WOSA formulation. Section III derives a discrete-time formulation for the TWCOH estimator and the corresponding WOSA cross-spectral and spectral estimators. The 
validity ranges for scale and time are carefully formulated. In Section IV we recast the WOSA components into multitaper form, allowing a simple expression for the number of complex degrees of freedom. Section V defines, as in [6], bivariate complex processes and the three types of resultant wavelet coherence. Theorem 1 shows that all three types of TWCOH estimator asymptotically have Goodman's distribution, and importantly the corresponding number of complex degrees of freedom are known. The excellent fit of Goodman's distribution to simulated TWCOH values is seen in Section VI, which also illustrates the theoretical results on solar physics data. Some concluding comments are provided in Section VII.

\section{BACKGROUND}

\section{A. Smoothing With the Morlet Wavelet}

The Morlet "wavelet" $\psi(t)=C \mathrm{e}^{-(1 / 2)((t / d))^{2}} \mathrm{e}^{\mathrm{i} \omega_{0} t}, d>0$, is a complex sinusoid localized/tapered by a Gaussian envelope ([4], p. 139). In order that this function can be treated like a wavelet it must have an integral that differs trivially from zero. The choice $\omega_{0}=2 \pi$ ensures that the integral of the function is less that $10^{-4.4}$ for $0.8 \leq d \leq 10$. For this range of $d$ we, therefore, take $\psi(t)=C \mathrm{e}^{-(1 / 2)((t / d))^{2}} \mathrm{e}^{\mathrm{i} 2 \pi t}$. The choice $\omega_{0}=2 \pi$ was also made in [17, eq. 14]. $C$ is chosen such that the $L^{2}$ norm of $\psi(\cdot)$ is unity: $1=\int_{-\infty}^{\infty}|\psi(t)|^{2} \mathrm{~d} t=C^{2} \int_{-\infty}^{\infty} \mathrm{e}^{-((t / d))^{2}} \mathrm{~d} t=$ $C^{2} d \pi^{1 / 2}$, giving $C=d^{-1 / 2} \pi^{-1 / 4}$. So

$$
\psi(t)=d^{-1 / 2} \pi^{-1 / 4} \mathrm{e}^{\mathrm{i} 2 \pi t} \mathrm{e}^{-\frac{1}{2}\left(\frac{t}{d}\right)^{2}} .
$$

The unit norm Fourier transform turns out to be

$$
\Psi(f) \equiv \int_{-\infty}^{\infty} \psi(t) \mathrm{e}^{-\mathrm{i} 2 \pi f t} \mathrm{~d} t=\pi^{1 / 4}(2 d)^{1 / 2} \mathrm{e}^{-2[d \pi(f-1)]^{2}}
$$

which is real-valued, like the Morse wavelets used in [6]. Examples of $\psi(t)$ and $\Psi(f)$ for some small values of $d$ are given in Fig. 1. $\Psi(1)$ is the maximum value of $\Psi(f)$. For very large values of $d$ the wavelet analysis effectively becomes a Fourier analysis [17].

From (4) we can express the scaled $(|a|<\infty)$ and translated $(b \in \mathbb{R})$ version as

$$
\begin{aligned}
\psi_{a, b}(t) & =\pi^{-1 / 4}(|a| d)^{-1 / 2} \mathrm{e}^{-\frac{1}{2}\left(\frac{t-b}{a d}\right)^{2}} \mathrm{e}^{\mathrm{i} 2 \pi\left(\frac{t-b}{a}\right)} \\
& =\lambda(a, b, d ; t) \mathrm{e}^{\mathrm{i} 2 \pi\left(\frac{t-b}{a}\right)}
\end{aligned}
$$

where $\lambda(a, b, d ; t) \equiv \pi^{-1 / 4}(|a| d)^{-1 / 2} \mathrm{e}^{-(1 / 2)((t-b) /(a d))^{2}}$ is the Gaussian component of $\psi_{a, b}(t)$ with unit $L^{2}$ norm. This form of wavelet has a simplicity of interpretation: to each scale $a$ corresponds a frequency $1 / a,[17]$.

Using (5) the CWT in (1) can be expressed as

$$
W(a, b ; Z, \psi)=\mathrm{e}^{\mathrm{i} 2 \pi b / a} \int_{-\infty}^{\infty} Z(t) \lambda(a, b, d ; t) \mathrm{e}^{-\mathrm{i} 2 \pi t / a} \mathrm{~d} t
$$

an appealingly simple form which follows from the choice of wavelet.

By defining the function $T_{Z ; a, b}(t)$ to be the product of the process $Z(t)$ with the normalized Gaussian component of the wavelet, namely, $T_{Z ; a, b}(t)=Z(t) \lambda(a, b, d ; t)$, we see that
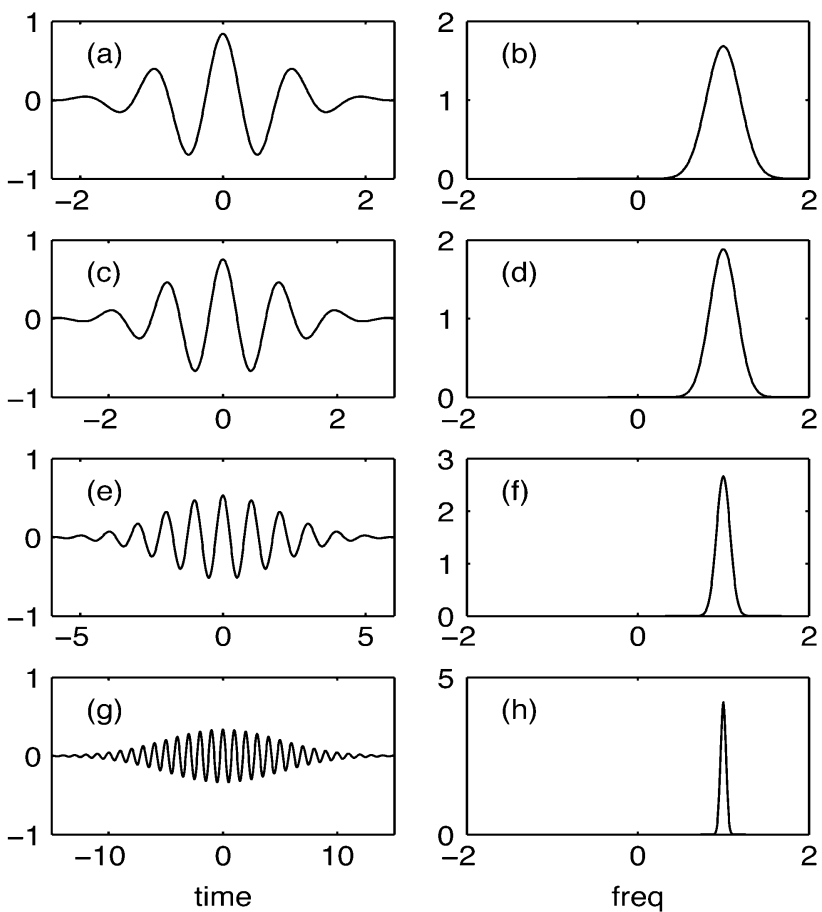

Fig. 1. The real part of the Morlet wavelet (left column) for (a) $d=0.8$, (c) $d=1$, (e) $d=2$, and (g) $d=5$, with the corresponding magnitude of the Fourier transform, $\Psi(f)$ in the right column. The wavelets are plotted over $[-3 d, 3 d]$ in each case.

$W(a, b ; Z, \psi)=\mathrm{e}^{\mathrm{i} 2 \pi b / a} \mathcal{T}_{Z ; a, b}(1 / a)$, where $\mathcal{T}_{Z ; a, b}(f)$ is the Fourier transform of the function $T_{Z ; a, b}(t)$, i.e.

$$
\mathcal{T}_{Z ; a, b}(f)=\int_{-\infty}^{\infty} Z(t) \lambda(a, b, d ; t) \mathrm{e}^{-\mathrm{i} 2 \pi f t} \mathrm{~d} t .
$$

Using this form (3) becomes

$$
\begin{aligned}
\mathcal{S}\left\{W\left(a, b ; Z_{1}, \psi\right)\right. & \left.W^{*}\left(a, b ; Z_{2}, \psi\right)\right\} \\
& =\frac{1}{\delta} \int_{b-\frac{\delta}{2}}^{b+\frac{\delta}{2}} \mathcal{T}_{Z_{1} ; a, \tau}(1 / a) \mathcal{T}_{Z_{2} ; a, \tau}^{*}(1 / a) \mathrm{d} \tau
\end{aligned}
$$

with analogous expressions for the denominator terms in (2). An expression similar to (7) in [13, p. 162] was recognized as a WOSA-type estimate, but few further details on the correspondence were given. In particular, we need to understand the continuous-time formulation of weighted overlapping segment averaging, which we now develop.

\section{B. A Continuous-Time WOSA Formulation}

For a stochastic process $Z(t)$, we split up a time interval $[b-$ $T / 2, b+T / 2]$ centred on $b$ into $N_{B}$ overlapping intervals of equal measure. The length of each subinterval is denoted by $\theta$. Each interval is offset from the previous one by $\Lambda=(T-$ $\theta) /\left(N_{B}-1\right)$. In this case the segment percentage overlap will be $100(1-\Lambda / \theta)$. We label the centre of the $j$ th interval as $t_{j}$ where $t_{j}=b-[(T-\theta) / 2]+j \Lambda$.

The $j$ th segment can be expressed as $Z(t) \chi_{I_{j}}(t)$, where $I_{j}=$ $[b-(T / 2)+j \Lambda, b-(T / 2)+\theta+j \Lambda]$, and $\chi_{[\alpha, \beta]}(t)$ is the characteristic function, namely $\chi_{[\alpha, \beta]}(t)=1$ if $\alpha \leq t \leq \beta$, and zero otherwise. 
For a continuous process we make use of a continuous data taper $h(t)$ of unit $L^{2}$ norm. Suppose $h(t)$ is designed to be compactly supported on the unit interval $[-1 / 2,1 / 2]$, we define $h_{\theta}(t) \equiv \theta^{-1 / 2} h(t / \theta), \theta>0$, which has unity $L^{2}$ norm on $[-\theta / 2, \theta / 2]$.

We can now define the $j$ th weighted Fourier transform $J_{Z ; j}(f)$ for the $j$ th signal segment centred at $t_{j}$ as $J_{Z ; j}(f)=\int_{-\infty}^{\infty} Z(t) \chi_{I_{j}} h_{\theta}\left(t-t_{j}\right) \mathrm{e}^{-\mathrm{i} 2 \pi t f} \mathrm{~d} t$. A WOSA cross-spectral estimator for $Z_{1}(t)$ and $Z_{2}(t)$ may be found by averaging over the $N_{B}$ individual segment estimates giving $\left(1 / N_{B}\right) \sum_{j=0}^{N_{B}-1} J_{Z_{1} ; j}(f) J_{Z_{2} ; j}^{*}(f) \equiv \hat{S}_{12, b}^{(W)}(f)$.

By letting $N_{B} \rightarrow \infty$ and $\Lambda \rightarrow 0$ such that $N_{B} \Lambda=(T-\theta)$

$$
\begin{aligned}
\hat{S}_{12, b}^{(W)}(f) & \rightarrow \lim _{\Lambda \rightarrow 0} \frac{\Lambda}{T-\theta} \sum_{j=0}^{N_{B}-1} J_{Z_{1} ; j}(f) J_{Z_{2} ; j}^{*}(f) \\
& =\frac{1}{T-\theta} \int_{b-\frac{T-\theta}{2}}^{b+\frac{T-\theta}{2}} J_{Z_{1} ; t^{\prime}}(f) J_{Z_{2} ; t^{\prime}}^{*}(f) \mathrm{d} t^{\prime}
\end{aligned}
$$

where

$$
J_{Z ; t^{\prime}}(f)=\int_{-\infty}^{\infty} Z(t) \chi_{\left[t^{\prime}-\frac{\theta}{2}, t^{\prime}+\frac{\theta}{2}\right]}(t) h_{\theta}\left(t-t^{\prime}\right) \mathrm{e}^{-\mathrm{i} 2 \pi f t} \mathrm{~d} t .
$$

This limiting form of weighted overlapping segment averaging gives an effective $100 \%$ overlap. If we set $\delta=T-\theta$ then (8) becomes $(1 / \delta) \int_{b-(\delta) / 2}^{b+(\delta) /(2)} J_{Z_{1} ; t^{\prime}}(f) J_{Z_{2} ; t^{\prime}}^{*}(f) \mathrm{d} t^{\prime}$, and the similarity to (7) is immediately apparent. We can be more precise. By making the approximation

$$
\mathrm{e}^{-\frac{1}{2}\left(\frac{t-\tau}{a d}\right)^{2}} \approx \mathrm{e}^{-\frac{1}{2}\left(\frac{t-\tau}{a d}\right)^{2}} \chi_{[\tau-3|a| d, \tau+3|a| d]}
$$

then from (6) we can express $\mathcal{T}_{Z ; a, \tau}(1 / a)$ as

$$
\int_{-\infty}^{\infty} Z(t) \chi_{[\tau-3|a| d, \tau+3|a| d]} \lambda(a, \tau, d ; t) \mathrm{e}^{-\mathrm{i} 2 \pi f t} \mathrm{~d} t .
$$

We note that this is in the form of (9) where $h(t)$ is a unit $L^{2}$ norm Gaussian-like function, $h(t) \equiv 6^{1 / 2} \pi^{-1 / 4} \mathrm{e}^{-(1 / 2)(6 t)^{2}}$, and $\theta \equiv 6|a| d$.

Putting (7) and (11) together we can conclude that $\mathcal{S}\left\{W\left(a, b ; Z_{1}, \psi\right) W^{*}\left(a, b ; Z_{2}, \psi\right)\right\}$ is equivalent to a limiting $100 \%$-overlap continuous-time WOSA cross-spectral estimator involving the signal portions $Z_{1}(t) \chi_{[\alpha, \beta]}$ and $Z_{2}(t) \chi_{[\alpha, \beta]}$ evaluated at the frequency $1 / a$ with $\alpha=b-[(\delta+\theta) / 2]=$ $b-(\delta / 2)-3|a| d$ and $\beta=b+[(\delta+\theta) / 2]=b+(\delta / 2)+3|a| d$.

\section{Choice of $\delta$}

We are left with the problem of choosing a suitable $\delta$ with which to control the size of the integration window. When performing temporal smoothing on wavelet transformations using the Morlet wavelet not only does the period of the sinusoid increase/decrease as $|a|$ increases/decreases but the width of the window, (the Gaussian-type component of the wavelet), also increases/decreases as a function of $|a|$. As a result $\delta$ must become a function of the scale $|a|$, meaning that for a translation $b$ the smoothing occurs over the interval $[b-(\delta(|a|)) / 2, b+$ $(\delta(|a|)) / 2]$.
For a specific choice of parameter $d$ the Morlet wavelet exhibits $\omega_{d}$ sinusoidal periods within the Gaussian envelope. We can say that $\omega_{d} \approx 6|a| d /|a|=6 d$ and hence is independent of $|a|$. The number of these sinusoidal oscillations that could be contained in an interval of length $\delta$ is $\kappa_{\delta}=\delta /|a|$. It is the value $\kappa_{\delta}$ that we wish to keep constant for all values $|a|$ and as such we let $\delta=|a| \kappa$, for a constant $\kappa>0$, giving

$$
\begin{aligned}
\mathcal{S}\left\{W\left(a, b ; Z_{1}, \psi\right)\right. & \left.W^{*}\left(a, b ; Z_{2}, \psi\right)\right\} \\
= & \frac{1}{|a| \kappa} \int_{b-\frac{|a| \kappa}{2}}^{b+\frac{|a| \kappa}{2}} \mathcal{T}_{Z_{1} ; a, \tau}(1 / a) \mathcal{T}_{Z_{2} ; a, \tau}^{*}(1 / a) \mathrm{d} \tau .
\end{aligned}
$$

\section{DisCRETE TIME METHOD}

\section{A. Smoothing in the Discrete Case}

Using (1), we firstly write $W(a, b ; Z, \psi)=\left[Z * \psi_{a}^{\prime}\right](b)$, where "*" denotes convolution and $\psi_{a}^{\prime}(t)=\psi_{a}^{*}(-t)$ and $\psi_{a}^{*}(t)=$ $|a|^{-1 / 2} \psi^{*}(t / a)$. Then since the Fourier transform of $\psi_{a}^{\prime}(t)$ is

$$
|a|^{-1 / 2} \int_{-\infty}^{\infty} \psi^{*}\left(\frac{-t}{a}\right) \mathrm{e}^{-\mathrm{i} 2 \pi f t} \mathrm{~d} t=|a|^{1 / 2} \Psi^{*}(a f)
$$

where $\Psi(f)$ is the Fourier transform of $\psi(t)$, we can express $W(a, b ; Z, \psi)$ in terms of the inverse Fourier transform:

$$
W(a, b ; Z, \psi)=|a|^{1 / 2} \int_{-\infty}^{\infty} \mathcal{Z}(f) \Psi^{*}(a f) \mathrm{e}^{\mathrm{i} 2 \pi f b} \mathrm{~d} f
$$

where $\mathcal{Z}(f)$ is the Fourier transform of $Z(t)$. Suppose

$$
|\mathcal{Z}(f)|=0 \text { for }|f|>f_{\mathcal{N}} \equiv 1 /[2 \Delta]
$$

for a sample interval $\Delta$. Then for a large, even, value of $N$, the CWT $W(a, b ; Z, \psi)$ can be approximated by

$$
\frac{|a|^{1 / 2}}{N \Delta} \sum_{q=-(N / 2)}^{(N / 2)-1} \mathcal{Z}\left(\frac{q}{N \Delta}\right) \Psi^{*}\left(a \frac{q}{N \Delta}\right) \mathrm{e}^{\mathrm{i} 2 \pi q b /(N \Delta)} .
$$

Let $Z_{n} \equiv Z(n \Delta), n=0, \ldots, N-1$, and let $a=a_{0} \Delta$ and $b=b_{0} \Delta, b_{0} \in \mathbb{Z}$. Then from (13) we have

$$
W(a, b ; Z, \psi) \approx \frac{\left|a_{0}\right|^{1 / 2}}{N(\Delta)^{1 / 2}} \sum_{q=0}^{N-1} \mathcal{Z}_{q} \Psi_{q}^{*}\left(a_{0}\right) \mathrm{e}^{\mathrm{i} 2 \pi q b_{0} / N}
$$

where $\mathcal{Z}_{q}=\Delta \sum_{n=0}^{N-1} Z_{n} \mathrm{e}^{-\mathrm{i} 2 \pi n q / N}$, for $q=0, \ldots, N-1$, the discrete Fourier transform of $\left\{Z_{t}\right\}$, and

$$
\Psi_{q}^{*}\left(a_{0}\right)= \begin{cases}\Psi^{*}\left(a_{0} \frac{q}{N}\right), & q=0, \ldots,(N / 2)-1 \\ \Psi^{*}\left(a_{0} \frac{q-N}{N}\right), & q=(N / 2), \ldots, N-1 .\end{cases}
$$

Hence

$$
W(a, b ; Z, \psi) \approx \frac{|a|^{1 / 2}}{N} \sum_{n=0}^{N-1} Z_{n} \sum_{q=0}^{N-1} \Psi_{q}^{*}\left(a_{0}\right) \mathrm{e}^{\mathrm{i} 2 \pi q\left(b_{0}-n\right) / N} .
$$


By making use of the form of (15) this may be rewritten as

$$
\begin{aligned}
& W(a, b ; Z, \psi) \\
& \approx \frac{|a|^{1 / 2}}{N} \sum_{n=0}^{N-1} Z_{n} \\
& \quad \times \sum_{q=-(N / 2)}^{(N / 2)-1} \Psi^{*}\left(a_{0} \frac{q}{N}\right) \mathrm{e}^{\mathrm{i} 2 \pi q\left(b_{0}-n\right) / N} \\
& \approx \Delta \sum_{n=0}^{N-1} Z_{n}|a|^{1 / 2} \int_{-f_{\mathcal{N}}}^{f_{\mathcal{N}}} \Psi^{*}(a f) \mathrm{e}^{\mathrm{i} 2 \pi f(b-n \Delta)} \mathrm{d} f .
\end{aligned}
$$

Provided

$$
|a| f_{\mathcal{N}}>f^{\prime}(d) \quad \text { where } \quad f^{\prime}(d) \equiv \max \left\{\left|f_{1}\right|,\left|f_{2}\right|\right\}
$$

and the effective support of $\Psi(f)$ is $\left[f_{1}, f_{2}\right]$, then

$$
\begin{aligned}
|a|^{1 / 2} \int_{-f_{\mathcal{N}}}^{f_{\mathcal{N}}} \Psi^{*}(a f) \mathrm{e}^{\mathrm{i} 2 \pi f(b-n \Delta)} \mathrm{d} f \\
=|a|^{1 / 2} \int_{-\infty}^{\infty} \Psi^{*}(a f) \mathrm{e}^{\mathrm{i} 2 \pi f(b-n \Delta)} \mathrm{d} f \\
=\frac{1}{|a|^{1 / 2}} \psi^{*}([n \Delta-b] / a)=\psi_{a, b}^{*}(n \Delta) .
\end{aligned}
$$

From (16) and (18), we take the discretized form of the CWT for our analysis to be defined by $W(a, b ; Z, \psi) \equiv \Delta \sum_{n=0}^{N-1} Z_{n} \psi_{a, b}^{*}(n \Delta)$. The form of $\psi_{a, b}(\cdot)$ used is given by (5). So $W(a, b ; Z, \psi)=\Delta \sum_{n=0}^{N-1} Z_{n} \lambda_{a, b, d ; n} \mathrm{e}^{-\mathrm{i} 2 \pi((n \Delta-b) /(a))}$, where $\lambda_{a, b, d ; n} \equiv \lambda(a, b, d ; n \Delta)$ is a taper for which $\Delta \sum_{n=-\infty}^{\infty} \lambda_{a, b, d ; n}^{2}=1$. Let $T_{Z ; a, b, n}=Z_{n} \lambda_{a, b, d ; n}$. Then

$$
W(a, b ; Z, \psi)=\mathrm{e}^{\mathrm{i} 2 \pi b / a} \Delta \sum_{n=0}^{N-1} T_{Z ; a, b, n} \mathrm{e}^{-\mathrm{i} 2 \pi n \Delta / a} .
$$

Hence, $W(a, b ; Z, \psi)=\mathrm{e}^{\mathrm{i} 2 \pi b / a} \mathcal{T}_{Z ; a, b}(1 / a)$, where $\mathcal{T}_{Z ; a, b}(f)$ is the Fourier transform of $\left\{T_{Z ; a, b, n}, n=0, \ldots, N-1\right\}$, i.e., $\mathcal{T}_{Z ; a, b}(f) \equiv \Delta \sum_{n=0}^{N-1} T_{Z ; a, b, n} \mathrm{e}^{-\mathrm{i} 2 \pi f n \Delta}$.

We now consider smoothing for the discrete case, analogous to the continuous case. Given jointly stationary series $Z_{1}(t)$ and $Z_{2}(t)$ satisfying (12), for the smoothing we average over a set of $N_{B}=2 M+1$ discrete time indices in the neighborhood of the translation point of interest $b=b_{0} \Delta$,

$$
\begin{aligned}
\mathcal{S}\left\{W\left(a, b ; Z_{1}, \psi\right) W^{*}\left(a, b ; Z_{2}, \psi\right)\right\} \\
=\frac{1}{N_{B}} \sum_{l=b_{0}-M}^{b_{0}+M} W\left(a, l \Delta ; Z_{1}, \psi\right) W^{*}\left(a, l \Delta ; Z_{2}, \psi\right) \\
=\frac{1}{N_{B}} \sum_{l=b_{0}-M}^{b_{0}+M} \mathcal{T}_{Z_{1} ; a, l \Delta}(1 / a) \mathcal{T}_{Z_{2} ; a, l \Delta}^{*}(1 / a) .
\end{aligned}
$$

Now $\mathcal{T}_{Z_{1} ; a, l \Delta}(\cdot)$ is the frequency domain function calculated on the segment of $\left\{Z_{1, n}\right\}$ centred at time index $l$ and tapered by the Gaussian taper. Then (20) is a WOSA cross-spectral estimator involving the signal portions $\left\{\tilde{Z}_{1, n}, n=0, \ldots, N_{P}-1\right\}$
TABLE I

COMPARISON OF PARAMETERS IN CONTINUOUS-TIME AND DISCRETE-TIME CASES

\begin{tabular}{|c||c|c|}
\hline & Cont. & Disc. \\
\hline \hline tapering & $\theta=6|a| d$ & $N_{S}=2 \nu+1 ; \nu=\left\lceil 3\left|a_{0}\right| d\right\rceil$ \\
\hline smoothing & $\delta=|a| \kappa$ & $N_{B}=2 M+1 ; M=\left\lceil\left|a_{0}\right| \kappa_{0}\right\rceil$ \\
\hline block size & $T=\delta+\theta$ & $N_{P}=N_{B}+N_{S}-1$ \\
\hline
\end{tabular}

and $\left\{\tilde{Z}_{2, n}, n=0, \ldots, N_{P}-1\right\}$ evaluated at frequency $1 / a$ where

$$
\tilde{Z}_{m, n} \equiv Z_{m,\left(b_{0}-\frac{N_{S}-1}{2}-M+n\right)}
$$

for $m=1,2$, and $N_{P}=N_{B}+N_{S}-1$, with $N_{S}$ assumed odd and equal to the number of terms included by the Gaussian taper. We shall refer to $N_{P}$ as the 'block size.' In view of (10) the support of the dimensionless taper

$$
\lambda_{n}^{0} \equiv \pi^{-1 / 4}\left(\left|a_{0}\right| d\right)^{-1 / 2} \exp \left(-\left[\left(n-b_{0}\right) /\left(a_{0} d\right)\right]^{2} / 2\right)
$$

is $n \in\left[b_{0}-\left\lceil 3\left|a_{0}\right| d\right\rceil, b_{0}+\left\lceil 3\left|a_{0}\right| d\right\rceil\right]$, where $\lceil x\rceil$ denotes the smallest integer exceeding $x$. Let $\nu \equiv\left[3\left|a_{0}\right| d\right]$. For this taper $\sum_{b_{0}-\nu}^{b_{0}+\nu}\left(\lambda_{n}^{0}\right)^{2}=1$, and it follows that $N_{S}=2 \nu+1$.

It is convenient to define a shifted version of the taper

$$
g_{n} \equiv \lambda_{n-\nu+b_{0}}^{0}, \quad n=0, \ldots, N_{S}-1 .
$$

The offset in the WOSA scheme is a single sample point so the percentage overlap is $100\left(1-1 / N_{S}\right) \%$. As for the continuous case, we should choose $M$ proportional to $|a|$.

\section{B. Parameters in Continuous and Discrete Cases}

Table I compares the definitions of the relevant parameters in the continuous-time and discrete-time cases. Notice that the block size $N_{P}$ is a function of $\left|a_{0}\right|, d$ and $\kappa_{0}$.

\section{Restrictions on $a$ and $b$}

The minimum usable absolute scale is specified by (17)

$$
|a|=a_{\min }(d)=f^{\prime}(d) / f_{\mathcal{N}} .
$$

We determine the cut-off frequency $f^{\prime}(d)$ as the lowest frequency for which $\Psi(f)<10^{-6} \times \Psi(1)$ for all $f>f^{\prime}(d)$.

Consider a specific positive $a>a_{\min }$. For any $b_{0}$ the Gaussian taper will extend from $b_{0}-\nu$ to $b_{0}+\nu$ (see Table I). The data extends from 0 to $N-1$, so if we are to avoid making assumptions such as circularity, we will only be able to accurately calculate $W(a, b ; Z, \psi)$ for $\nu \leq b_{0} \leq N-1-\nu$. In view of the averaging in (20) this must be further modified for the calculation of $\mathcal{S}\left\{W\left(a, b ; Z_{1}, \psi\right) W^{*}\left(a, b ; Z_{2}, \psi\right)\right\}$ to $\nu+M(a) \leq b_{0}(a) \leq N-1-\nu-M(a)$. Let

$$
b_{\min }(a)=\nu+M(a) ; b_{\max }(a)=N-1-\nu-M(a) .
$$

Note that for $b_{\min }(a)$, the signal portion, namely, $\left\{\tilde{Z}_{m, n} ; n=\right.$ $\left.0, \ldots, N_{P}-1\right\}$, is $\left\{Z_{m, 0}, \ldots, Z_{m, N_{P}-1}\right\}$, and for $\left\{b_{\max }(a)\right\}$ the signal portion is $\left\{Z_{m, N-N_{P}}, \ldots, Z_{m, N-1}\right\}$, for $m=1,2$, 

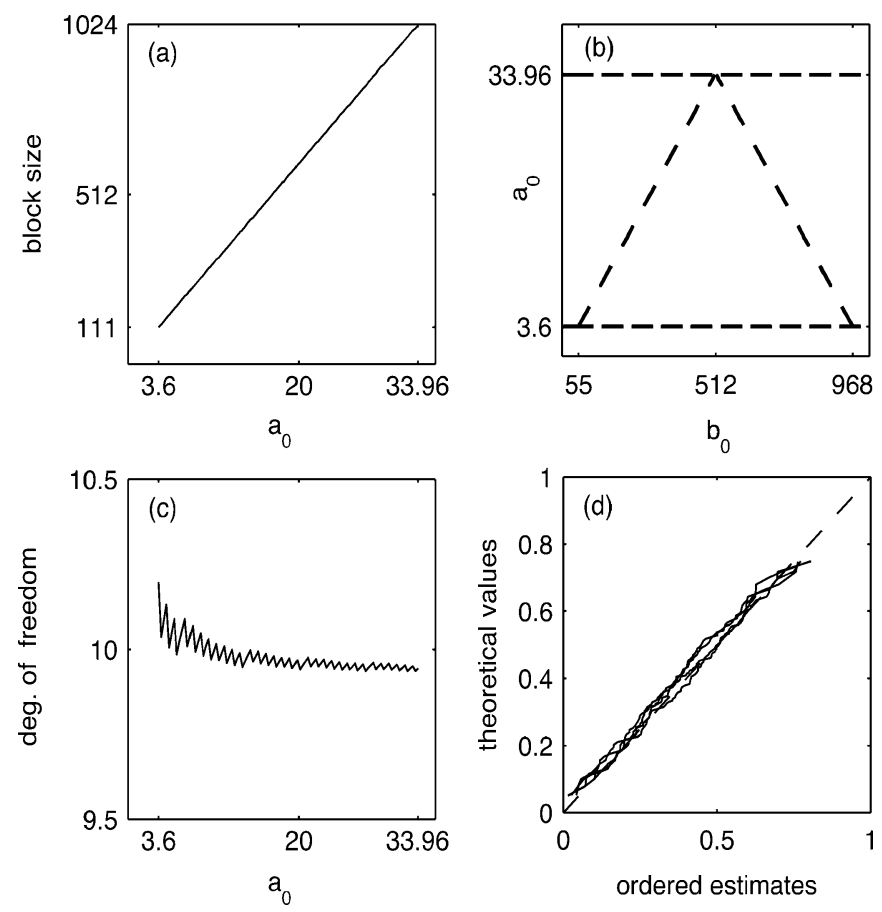

Fig. 2. Parameter relationships and statistical goodness-of-fit assessment when $d=1, f^{\prime}(d)=1.8, N=1024, \kappa_{0}=12$. (a) Block size $N_{P}$ versus $a_{0}$. (b) Validity region for $a_{0}, b_{0}$ given by interior of central triangle. (c) Degrees of freedom $\varphi$ versus $a_{0}$. (d) Goodman Q-Q plots for four different $\left(a_{0}, b_{0}\right)$ locations.

so there are no end-effects, as expected. We must have $b_{\min }(a) \leq b_{\max }(a)$,

$$
\begin{aligned}
\nu+M(a) & \leq N-1-\nu-M(a) \\
N_{S}+2 M(a) & \leq N
\end{aligned}
$$

i.e., $N_{S}+N_{B}-1 \leq N$, or $N_{P} \leq N$, which merely says that the block size, $N_{P}$, used in calculating a single point in the WOSA-type estimator must be less than or equal to the total number of observed data points, $N$, as is obviously required. So the range for $b_{0}$ is specified by (24).

Finally, we must determine the maximum absolute analysis scale $a_{\max }$. From (25)

$$
\left\lceil 3\left|a_{0}\right| d\right\rceil+\left\lceil\left|a_{0}\right| \kappa_{0}\right\rceil \leq(N-1) / 2 .
$$

But since $\lceil x y\rceil=x y+\xi, 0 \leq \xi<1$, (26) can be written $3\left|a_{0}\right| d+\left|a_{0}\right| \kappa_{0} \leq[(N-1) / 2]-\xi_{0}$, for $0 \leq \xi_{0}<2$. Hence, $a_{\max }$ is given by

$$
a_{\max }=\Delta \frac{[(N-1) / 2]-2}{3 d+\kappa_{0}}=\Delta \frac{N-5}{6 d+2 \kappa_{0}} .
$$

The variation of block size $N_{P}$ for positive $a_{0}$ with $a_{\min } / \Delta \leq$ $a_{0} \leq a_{\max } / \Delta$ is given in Figs. 2(a) and 3(a) for $d=1, f^{\prime}(d)=$ $1.8, N=1024, \kappa_{0}=12$ and $d=2, f^{\prime}(d)=1.4, N=$ $1024, \kappa_{0}=24$, respectively. The interiors of the central triangles of Figs. 2(b) and 3(b) show the corresponding valid regions for $a_{0}, b_{0}$. The equivalent for negative $a_{0}$ follows by reflection about $a_{0}=0$.
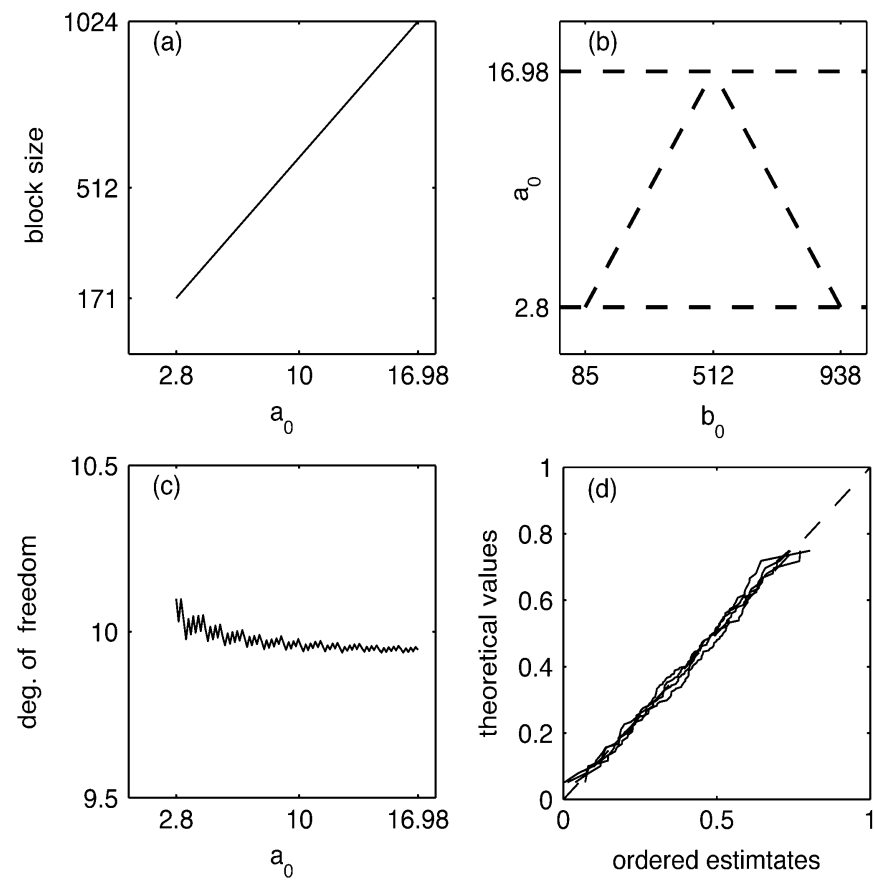

Fig. 3. Same style of plots as for Fig. 2 but with $d=2, f^{\prime}(d)=1.4, N=$ $1024, \kappa_{0}=24$.

\section{Review}

When using the Morlet wavelet, temporal smoothing is mathematically representable as a form of WOSA estimation based on a subsection of the processes of block size $N_{P}$. The overlap is $100\left(1-1 / N_{S}\right) \%$. If we know the distributional properties of such WOSA spectral estimators, and magnitude-squared coherence estimators (MSCOH) formed from them via (2), then we can determine the distributional properties of TWCOH estimators.

\section{Statistical Properties of WOSA CROSS-SPECTRAL ESTIMATORS}

\section{A. The TWCOH Estimator}

For the estimator (20) define

$$
\hat{S}_{12, b}(1 / a) \equiv \mathcal{S}\left\{W\left(a, b ; Z_{1}, \psi\right) W^{*}\left(a, b ; Z_{2}, \psi\right)\right\}
$$

where $\hat{S}_{12, b}(f)$ is a WOSA cross-spectral estimator involving the signal portions $\left\{\tilde{Z}_{1, n}, n=0, \ldots, N_{P}-1\right\}$ and $\left\{\tilde{Z}_{2, n}, n=\right.$ $\left.0, \ldots, N_{P}-1\right\}$ evaluated at frequency $1 / a$ where $\tilde{Z}_{m, n}$ is given in (21). The offset is unity and $N_{P}=N_{B}+N_{S}-1, N_{B}=$ $2 M+1$ and $N_{S}=2 \nu+1$.

Consider the construction of a WOSA cross-spectral estimator. The block of size $N_{P}$ is divided into $N_{B}$ subblocks each of size $N_{S}$. A shift of unity is applied between successive subblocks. Using the data taper $g_{0}, \ldots, g_{N_{S}-1},(22)$, scaled so that $\sum_{n=0}^{N_{S}-1} g_{n}^{2}=1$, the WOSA cross-spectrum estimator is defined by

$$
\hat{S}_{12, b}(f) \equiv \frac{1}{N_{B}} \sum_{j=0}^{N_{B}-1} \hat{S}_{12, b ; j}(f)
$$


where the estimator for subblock $j,\left(0 \leq j \leq N_{B}-1\right)$, is $\hat{S}_{12, b ; j}(f)$, given by

$$
\Delta\left[\sum_{l=0}^{N_{S}-1} g_{l} \tilde{Z}_{1, l+j} \mathrm{e}^{-\mathrm{i} 2 \pi f l \Delta}\right]\left[\sum_{n=0}^{N_{S}-1} g_{n} \tilde{Z}_{2, n+j}^{*} \mathrm{e}^{\mathrm{i} 2 \pi f n \Delta}\right] .
$$

\section{B. Multitaper Form of WOSA Cross-Spectral Estimation}

The $j=0$ subblock utilizes data for times $0, \ldots, N_{S}-1$ while the final $j=N_{B}-1$ subblock uses data for times $N_{P}-$ $N_{S}, \ldots, N_{P}-1$. We can rewrite the estimator for subblock $j$ as [3]

$$
\Delta\left[\sum_{l=0}^{N_{P}-1} g_{j, l}^{\prime} \tilde{Z}_{1, l} \mathrm{e}^{-\mathrm{i} 2 \pi f l \Delta}\right]\left[\sum_{n=0}^{N_{P}-1} g_{j, n}^{\prime} \tilde{Z}_{2, n}^{*} \mathrm{e}^{\mathrm{i} 2 \pi f n \Delta}\right]
$$

where for $n=0, \ldots, N_{P}-1$

$$
g_{j, n}^{\prime}= \begin{cases}g_{n-j}, & \text { if } n=j, \ldots, j+N_{S}-1 \\ 0, & \text { otherwise }\end{cases}
$$

Hence, $\hat{S}_{12, b}(f)$ can be written as

$$
\frac{\Delta}{N_{B}} \sum_{j=0}^{N_{B}-1}\left[\sum_{l=0}^{N_{P}-1} g_{j, l}^{\prime} \tilde{Z}_{1, l} \mathrm{e}^{-i 2 \pi f l \Delta}\right]\left[\sum_{n=0}^{N_{P}-1} g_{j, n}^{\prime} \tilde{Z}_{2, n}^{*} \mathrm{e}^{\mathrm{i} 2 \pi f n \Delta}\right] .
$$

In fact we are able to manipulate this equation so that we are using a set of $N_{B}$ orthogonal tapers [27]. Let $D_{m, l}=\tilde{Z}_{m, l}^{*} \exp (\mathrm{i} 2 \pi f l \Delta)$, for $m=1,2$, and $\boldsymbol{D}_{m}=\left[D_{m, 0}, \ldots, D_{m, N_{P}-1}\right]^{T}$, where ${ }^{T}$ denotes transpose. Then

$$
\hat{S}_{12, b}(f)=\Delta \boldsymbol{D}_{1}^{H} \boldsymbol{B} \boldsymbol{B}^{T} \boldsymbol{D}_{2}
$$

where $\boldsymbol{B}$ is an $N_{P} \times N_{B}$ real matrix with $j$ th column given by $g_{j, n}^{\prime} / \sqrt{N_{B}}, n=0, \ldots, N_{P}-1$, and ${ }^{H}$ denotes Hermitian (complex conjugate) transpose.

The $N_{P} \times N_{P}$ outer product matrix $B B^{T}$ is symmetric positive semidefinite since $\hat{S}_{11, b}=\Delta \boldsymbol{D}_{1}^{H} \boldsymbol{B} \boldsymbol{B}^{T} \boldsymbol{D}_{1} \geq 0$. Hence [25, p. 296] $B B^{T}$ will have real-valued eigenvectors and nonnegative eigenvalues.

By choosing $\left\{g_{n}\right\}$ to have all nonzero elements the columns of $\boldsymbol{B}$ are linearly independent and so $\boldsymbol{B}$ is full-rank, namely $\operatorname{rank}\{\boldsymbol{B}\}=N_{B}$. Further to this, with real-valued $\left\{g_{n}\right\}$, $\operatorname{rank}\{\boldsymbol{B}\}=\operatorname{rank}\left\{\boldsymbol{B}^{T} \boldsymbol{B}\right\}=\operatorname{rank}\left\{\boldsymbol{B} \boldsymbol{B}^{T}\right\}$ and, hence, $\boldsymbol{B} \boldsymbol{B}^{T}$ is itself of rank $N_{B}$. From this we can determine that the matrix $B B^{T}$ has $N_{B}$ positive eigenvalues ordered in decreasing size $\sigma_{0} \geq \cdots \geq \sigma_{N_{B}-1}$ and the remainder zero, i.e., $\sigma_{N_{B}}, \ldots, \sigma_{N_{P}-1}=0$, with a set of corresponding orthonormal eigenvectors $\boldsymbol{u}_{0}, \ldots, \boldsymbol{u}_{N_{B}-1} \in \mathbb{R}^{N}$. We can write $\boldsymbol{B} \boldsymbol{B}^{T}$ in terms of the eigenvectors and eigenvalues $\boldsymbol{B B}^{T}=\boldsymbol{U} \boldsymbol{\Sigma} \boldsymbol{U}^{T}=\sum_{j=0}^{N_{B}-1} \sigma_{j} \boldsymbol{u}_{j} \boldsymbol{u}_{j}^{T}$ where $\boldsymbol{U}$ is an $N_{P} \times N_{B}$ matrix with $j$ th column $\boldsymbol{u}_{j}$ and $\boldsymbol{\Sigma}=\operatorname{diag}\left\{\sigma_{0}, \ldots, \sigma_{N_{B}-1}\right\}$.

We are able to equivalently write $\boldsymbol{U} \boldsymbol{\Sigma} \boldsymbol{U}^{T}=\boldsymbol{A} \boldsymbol{A}^{T}$ where $\boldsymbol{A}=\left[\boldsymbol{u}_{0} \sqrt{\sigma_{0}}, \ldots, \boldsymbol{u}_{N_{B}-1} \sqrt{\sigma}_{N_{B}-1}\right]$ where $\boldsymbol{A}$ is an $N_{P} \times N_{B}$ matrix with orthogonal columns. We can now write the WOSA spectral estimator in (30) as $\hat{S}_{12, b}(f)=\Delta \boldsymbol{D}_{1}^{H} \boldsymbol{A}^{T} \boldsymbol{D}_{2}$, i.e.

$$
\begin{aligned}
\hat{S}_{12, b}(f)=\frac{\Delta}{N_{B}} \sum_{j=0}^{N_{B}-1} \gamma_{j} & {\left[\sum_{l=0}^{N_{P}-1} u_{j, l} \tilde{Z}_{1, l} \mathrm{e}^{-\mathrm{i} 2 \pi f l \Delta}\right] } \\
& \times\left[\sum_{n=0}^{N_{P}-1} u_{j, n} \tilde{Z}_{2, n} \mathrm{e}^{-\mathrm{i} 2 \pi f n \Delta}\right]
\end{aligned}
$$

where $u_{j, l}$ is the $l$ th element of the vector $\boldsymbol{u}_{j}$ and $\gamma_{j}$ is the $j$ th weighting factor related to the $j$ th eigenvalue via $\gamma_{j}=N_{B} \sigma_{j}$. The standardization of $\left\{g_{n}\right\}$ means that $\sum_{j=0}^{N_{B}-1} \gamma_{j}=N_{B}$, [27]. The representation of $\hat{S}_{12, b}(f)$ in (31) is a weighted multitaper cross-spectral estimator that uses a set of $N_{B}$ orthonormal tapers to create a set of spectral estimators across which averaging can be carried out. A multitaper formulation for the spectrum $\hat{S}_{11, b}(1 / a) \equiv \mathcal{S}\left\{W\left(a, b ; Z_{1}, \psi\right) W^{*}\left(a, b ; Z_{1}, \psi\right)\right\}$, and likewise $\hat{S}_{22, b}(1 / a)$, follow simply by setting both time series to be the same.

In Section V we also have reason to consider

$$
\hat{R}_{12, b}(1 / a) \equiv \mathcal{S}\left\{W\left(a, b ; Z_{1}, \psi\right) W^{*}\left(a, b ; Z_{2}^{*}, \psi\right)\right\}
$$

where the second process is conjugated and

$$
\begin{aligned}
& \hat{R}_{12, b}(f)=\frac{\Delta}{N_{B}} \sum_{j=0}^{N_{B}-1} \gamma_{j} {\left[\sum_{l=0}^{N-1} u_{j, l} \tilde{Z}_{1, l} \mathrm{e}^{-\mathrm{i} 2 \pi f l \Delta}\right] } \\
& \times\left[\sum_{n=0}^{N-1} u_{j, n} \tilde{Z}_{2, n}^{*} \mathrm{e}^{-\mathrm{i} 2 \pi f n \Delta}\right]^{*} .
\end{aligned}
$$

\section{Degrees of Freedom}

It can be shown [27] that the estimator (31) consisting of $N_{B}$ orthonormal tapers with corresponding weighting factors $\gamma_{0}, \ldots, \gamma_{N_{B}-1}$ has

$$
\varphi=\frac{N_{B}^{2}}{\sum_{j=0}^{N_{B}-1} \gamma_{j}^{2}}=\frac{1}{\sum_{j=0}^{N_{B}-1} \sigma_{j}^{2}}
$$

complex degrees of freedom. The quantity $\varphi$ in (34) is critical in determining the distribution of TWCOH. Contours of constant degrees of freedom $\varphi$ as a function of $N_{S}$ and $N_{B}$ are shown in Fig. 4. Clearly, $\varphi$ is constant for a constant gradient or ratio $N_{B} / N_{S}$. But from Table I we see that for the sorts of values used in practice $N_{B} / N_{S} \approx \kappa_{0} /(3 d)$. For example for $\varphi=10, N_{B} / N_{S}=200 / 50=4 \approx \kappa_{0} /(3 d)$, so the choices $\kappa_{0}=12, d=1$ or $\kappa_{0}=24, d=2$, are both associated with approximately ten complex degrees of freedom. The use of this result is illustrated in Section VI.

\section{Alternative Approaches}

A recent study of the magnitude-squared ordinary coherence estimator was carried out by [1]. When using overlapping its distribution was assumed to be the same as in the nonoverlapped case, but with the number of degrees of freedom being different. A deterministic formula was derived for the degrees of freedom 


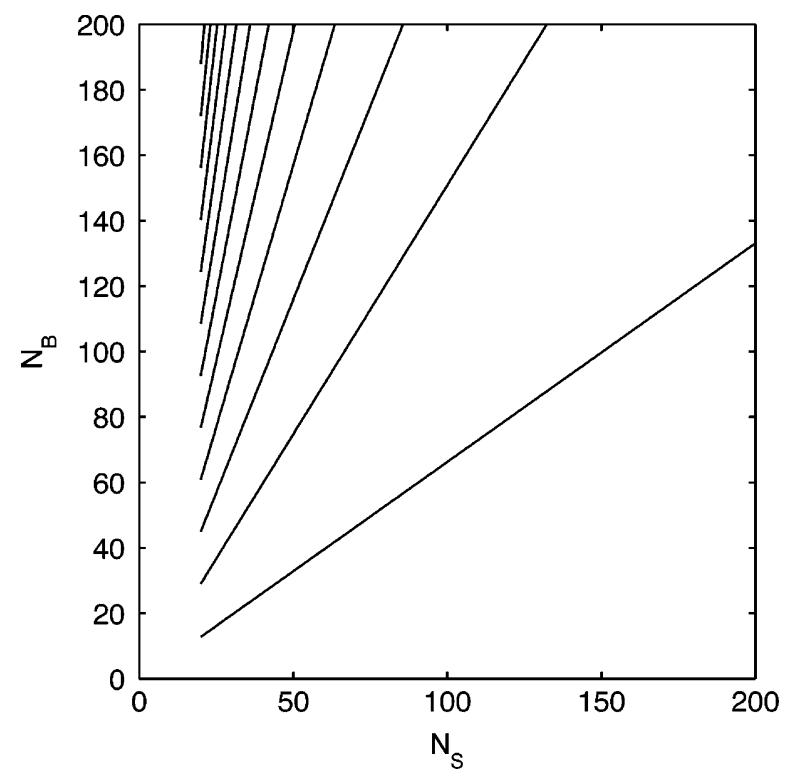

Fig. 4. Contours of constant degrees of freedom $\varphi$ as a function of $N_{S}$ and $N_{B}$. The lines start at $\varphi=2$ (lower right) and increase in steps of 2 towards the upper left.

from simulation results using a Hamming taper. Simulation was also used in [13] to assess the effect of overlapping on the bias and variance of coherence.

By way of contrast, the multitaper formulation delivers the basic statistical structure immediately. The degrees of freedom are given simply by (34), (a formula which is valid for different tapers). Further, Goodman's distribution follows from the form of the cross-spectral estimator (31), as discussed in the proof of Theorem 1.

\section{Statistical Analysis of WaVElet CoHEREnCE ESTIMATORS FOR COMPLEX GAUSSIAN PROCESSES}

Since the Morlet wavelet is complex-valued, we consider wavelet coherence for the most general case of complex-valued time series [6], [24].

\section{A. Bivariate Complex Processes}

Let $\left\{\boldsymbol{Z}_{n} \equiv\left[Z_{1, n}, Z_{2, n}\right]^{T}, n \in \mathbb{Z}\right\}$ be a zero mean complex-valued bivariate random process. Assume the two processes are jointly second-order stationary (SOS). We define cross-covariance sequences $s_{l m, \tau}=\operatorname{cov}\left\{Z_{l, n+\tau}, Z_{m, n}\right\}=$ $E\left\{Z_{l, n+\tau} Z_{m, n}^{*}\right\}$. The covariance between $Z_{l, n+\tau}$ and the conjugate of $Z_{m, n}$ is called the cross-relation between $Z_{l, n+\tau}$ and $Z_{m, n}$ and denoted by $\operatorname{rel}\left\{Z_{l, n+\tau}, Z_{m, n}\right\}$, i.e., $r_{l m, \tau}=\operatorname{rel}\left\{Z_{l, n+\tau}, Z_{m, n}\right\}=E\left\{Z_{l, n+\tau} Z_{m, n}\right\}$. Because the processes are SOS, $\operatorname{cov}\left\{Z_{l, n+\tau}, Z_{m, n}\right\}$ and $\operatorname{rel}\left\{Z_{l, n+\tau}, Z_{m, n}\right\}$, $l, m=1,2$, are functions of $\tau$ only.

We now append the conjugate bivariate process to obtain $\left\{\check{Z}_{n}=\left[Z_{1, n}, Z_{2, n}, Z_{1, n}^{*}, Z_{2, n}^{*}\right]^{T}, n \in \mathbb{Z}\right\}$. The corresponding lag- $\tau$ covariance matrix, $\boldsymbol{\Xi}_{\tau}=E\left\{\check{\boldsymbol{Z}}_{n+\tau} \check{\boldsymbol{Z}}_{n}^{H}\right\}$, is

$$
\boldsymbol{\Xi}_{\tau}=\left[\begin{array}{ll}
E\left\{\boldsymbol{Z}_{n+\tau} \boldsymbol{Z}_{n}^{H}\right\} & E\left\{\boldsymbol{Z}_{n+\tau} \boldsymbol{Z}_{n}^{T}\right\} \\
E\left\{\boldsymbol{Z}_{n+\tau}^{*} \boldsymbol{Z}_{n}^{H}\right\} & E\left\{\boldsymbol{Z}_{n+\tau}^{*} \boldsymbol{Z}_{n}^{T}\right\}
\end{array}\right]=\left[\begin{array}{cc}
\boldsymbol{s}_{\tau} & \boldsymbol{r}_{\tau} \\
\boldsymbol{r}_{\tau}^{*} & \boldsymbol{s}_{\tau}^{*}
\end{array}\right]
$$

and $\boldsymbol{s}_{\tau}$ and $\boldsymbol{r}_{\tau}$ are the $2 \times 2$ matrices $\boldsymbol{s}_{\tau}=E\left\{\boldsymbol{Z}_{n+\tau} \boldsymbol{Z}_{n}^{H}\right\}$ and $\boldsymbol{r}_{\tau}=E\left\{\boldsymbol{Z}_{n+\tau} \boldsymbol{Z}_{n}^{T}\right\}$, for which $\boldsymbol{s}_{\tau}=\boldsymbol{s}_{-\tau}^{H}$ and $\boldsymbol{r}_{\tau}=\boldsymbol{r}_{-\tau}^{T}$. The spectral matrix $\boldsymbol{\Upsilon}(f)=\Delta \sum_{\tau=-\infty}^{\infty} \boldsymbol{\Xi}_{\tau} \mathrm{e}^{-\mathrm{i} 2 \pi f \tau \Delta}$ of $\left\{\check{\boldsymbol{Z}}_{n}\right\}$ is given by

$$
\begin{aligned}
\boldsymbol{\Upsilon}(f) & =\left[\begin{array}{ccc}
\boldsymbol{S}(f) & \boldsymbol{R}(f) \\
\boldsymbol{R}^{*}(-f) & \boldsymbol{S}^{*}(-f)
\end{array}\right] \\
& =\left[\begin{array}{cccc}
S_{11}(f) & S_{12}(f) & R_{11}(f) & R_{12}(f) \\
S_{21}(f) & S_{22}(f) & R_{21}(f) & R_{22}(f) \\
R_{11}^{*}(-f) & R_{12}^{*}(-f) & S_{11}^{*}(-f) & S_{12}^{*}(-f) \\
R_{21}^{*}(-f) & R_{22}^{*}(-f) & S_{21}^{*}(-f) & S_{22}^{*}(-f)
\end{array}\right]
\end{aligned}
$$

where we note that $\boldsymbol{R}^{*}(-f)=\boldsymbol{R}^{H}(f)$ and $S^{*}(-f)=S^{T}(-f)$ since $\boldsymbol{S}(f)=\boldsymbol{S}^{H}(f)$ and $\boldsymbol{R}(f)=\boldsymbol{R}^{T}(-f)$.

Off-diagonal terms are cross-spectra. $S_{12}(f)$ is the conventional cross-spectrum of $\left\{Z_{1, t}\right\}$ and $\left\{Z_{2, t}\right\} . R_{12}(f)$ is the crossspectrum of $\left\{Z_{1, t}\right\}$ and $\left\{Z_{2, t}^{*}\right\}$, while $R_{11}(f)$ is the cross-spectrum of $\left\{Z_{1, t}\right\}$ with its conjugate $\left\{Z_{1, t}^{*}\right\} . R_{11}(f)$ and $R_{12}(f)$ are examples of relational cross-spectra [23].

For ease of notation we will henceforth write $\mathcal{S}\left\{W\left(a, b ; Z_{1}, \psi\right) W^{*}\left(a, b ; Z_{2}, \psi\right)\right\} \quad$ as $\quad \mathcal{S}\left\{Z_{1}, Z_{2}\right\}, \quad$ in particular, dependence on $a, b$ and $\psi$ is implicit. Consider the matrix $\hat{\mathbf{\Omega}}(a, b)$ given by

$\hat{\mathbf{\Omega}}(a, b)=$

$$
\left[\begin{array}{llll}
\mathcal{S}\left\{Z_{1}, Z_{1}\right\} & \mathcal{S}\left\{Z_{1}, Z_{2}\right\} & \mathcal{S}\left\{Z_{1}, Z_{1}^{*}\right\} & \mathcal{S}\left\{Z_{1}, Z_{2}^{*}\right\} \\
\mathcal{S}\left\{Z_{2}, Z_{1}\right\} & \mathcal{S}\left\{Z_{2}, Z_{2}\right\} & \mathcal{S}\left\{Z_{2}, Z_{1}^{*}\right\} & \mathcal{S}\left\{Z_{2}, Z_{2}^{*}\right\} \\
\mathcal{S}\left\{Z_{1}^{*}, Z_{1}\right\} & \mathcal{S}\left\{Z_{1}^{*}, Z_{2}\right\} & \mathcal{S}\left\{Z_{1}^{*}, Z_{1}^{*}\right\} & \mathcal{S}\left\{Z_{1}^{*}, Z_{2}^{*}\right\} \\
\mathcal{S}\left\{Z_{2}^{*}, Z_{1}\right\} & \mathcal{S}\left\{Z_{2}^{*}, Z_{2}\right\} & \mathcal{S}\left\{Z_{2}^{*}, Z_{1}^{*}\right\} & \mathcal{S}\left\{Z_{2}^{*}, Z_{2}^{*}\right\}
\end{array}\right]
$$

Using the definitions in (28) and (32), we see that this matrix can be equivalently written as $\hat{\Upsilon}_{b}(1 / a)$ given by

$$
\left[\begin{array}{cccc}
\hat{S}_{11, b}(1 / a) & \hat{S}_{12, b}(1 / a) & \hat{R}_{11, b}(1 / a) & \hat{R}_{12, b}(1 / a) \\
\hat{S}_{21, b}(1 / a) & \hat{S}_{22, b}(1 / a) & \hat{R}_{21, b}(1 / a) & \hat{R}_{22, b}(1 / a) \\
\hat{R}_{11, b}^{*}(-1 / a) & \hat{R}_{12, b}^{*}(-1 / a) & \hat{S}_{11, b}^{*}(-1 / a) & \hat{S}_{12, b}^{*}(-1 / a) \\
\hat{R}_{21, b}^{*}(-1 / a) & \hat{R}_{22, b}^{*}(-1 / a) & \hat{S}_{21, b}^{*}(-1 / a) & \hat{S}_{22, b}^{*}(-1 / a)
\end{array}\right] .
$$

(Obviously, $\hat{\boldsymbol{\Omega}}(a, b) \equiv \hat{\boldsymbol{\Upsilon}}_{b}(1 / a)$.) Equations (31) and (33) give the forms of the terms in this matrix. Note that $\hat{\Upsilon}_{b}(1 / a)$ is an estimator for $\Upsilon(1 / a)$ for different values of $b$; the $b$ merely defines which blocks of size $N_{P}$ of the time series are being used.

Theorem 1: Let $\left\{Z_{n}, n \in \mathbb{Z}\right\}$ be a zero mean complex-valued bivariate Gaussian SOS random process. Then, asymptotically

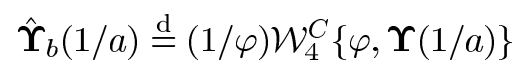

where $\mathcal{W}_{4}^{C}\{\varphi, \Upsilon(1 / a)\}$ denotes the four-dimensional complex central Wishart distribution with $\varphi$ complex degrees of freedom and mean $\varphi \Upsilon \Upsilon(1 / a)$.

Proof: This can be found in the Appendix.

Comparing (35) to the corresponding result in [6, eq. (29)], for the TWCOH approach used here the number of complex degrees of freedom is of the form (34), as opposed to the number of Morse wavelets used in [6]. But here the mapping between frequency, $f$, and scale, $a$, is simple: $f=1 / a$.

\section{B. Estimated Wavelet Coherencies}

For complex-valued processes three distinct wavelet coherencies of the form (2) can be defined. The first applies when the 
two signals are two different complex processes, the second for when the two signals are one process and the conjugate of the other, and the third when the two signals are a process and its own conjugate. (For real-valued signals only the first exists). In the frequency domain these three types of coherence are well established, see, e.g., [6] and [19]. The three estimators and true parameter values are

i) The ordinary TWCOH estimator $\hat{\gamma}^{2}(a, b)$ for $\left\{Z_{1, n}\right\}$ and $\left\{Z_{2, n}\right\}$, is given by

$$
\hat{\gamma}^{2}(a, b)=\left|\hat{\Omega}_{12}(a, b)\right|^{2} /\left[\hat{\Omega}_{11}(a, b) \hat{\Omega}_{22}(a, b)\right]
$$

with $\gamma^{2}(a, b)=\left|S_{12}(1 / a)\right|^{2} /\left[S_{11}(1 / a) S_{22}(1 / a)\right]$.

ii) The conjugate TWCOH estimator $\hat{\gamma}_{\dagger}^{2}(a, b)$ for $\left\{Z_{1, n}\right\}$ and $\left\{Z_{2, n}^{*}\right\}$, is

$$
\hat{\gamma}_{\dagger}^{2}(a, b)=\left|\hat{\Omega}_{14}(a, b)\right|^{2} /\left[\hat{\Omega}_{11}(a, b) \hat{\Omega}_{44}(a, b)\right]
$$

with $\gamma_{\dagger}^{2}(a, b)=\left|R_{12}(1 / a)\right|^{2} /\left[S_{11}(1 / a) S_{22}(-1 / a)\right]$.

iii) The conjugate TWCOH estimator $\hat{\gamma}_{*}^{2}(a, b)$ for $\left\{Z_{1, n}\right\}$ and $\left\{Z_{1, n}^{*}\right\}$, is given by

$$
\hat{\gamma}_{*}^{2}(a, b)=\left|\hat{\Omega}_{13}(a, b)\right|^{2} /\left[\hat{\Omega}_{11}(a, b) \hat{\Omega}_{33}(a, b)\right]
$$

with $\gamma_{*}^{2}(a, b)=\left|R_{11}(1 / a)\right|^{2} /\left[S_{11}(1 / a) S_{11}(-1 / a)\right]$. [Likewise, we would obtain a TWCOH estimator for $\left\{Z_{2, n}\right\}$ and $\left\{Z_{2, n}^{*}\right\}$ by using entries 2 and 4 of $\hat{\boldsymbol{\Omega}}(a, b)$ rather than 1 and 3 in (38).]

As a result of Theorem 1, all three coherencies have Goodman's distribution [9, eq. (5.54)]. Consider coherence of type (iii), the conjugate TWCOH estimator for $\left\{Z_{1, n}\right\}$ and $\left\{Z_{1, n}^{*}\right\}$. Its probability density function (pdf) is

$$
\begin{aligned}
g_{\hat{\gamma}_{*}^{2}}\left(x ; \varphi, \gamma_{*}^{2}\right)= & (\varphi-1)\left(1-\gamma_{*}^{2}\right)^{\varphi}(1-x)^{\varphi-2} \\
& \times_{2} F_{1}\left(\varphi, \varphi ; 1 ; \gamma_{*}^{2} x\right), \quad 0 \leq \hat{\gamma}_{*}^{2}<1
\end{aligned}
$$

where $\hat{\gamma}_{*}^{2} \equiv \hat{\gamma}_{*}^{2}(a, b)$ and $\gamma_{*}^{2} \equiv \gamma_{*}^{2}(a, b) \cdot{ }_{2} F_{1}\left(\alpha_{1}, \alpha_{2} ; \beta_{1} ; z\right)$ is the hypergeometric function with 2 and 1 parameters, $\alpha_{1}, \alpha_{2}$ and $\beta_{1}$, and scalar argument $z$ [10, p. 1045].

Note that if $\varphi<4$, the matrix $\hat{\boldsymbol{\Omega}}(a, b)$ will be singular. To obtain just one of the three possible TWCOH estimators, [such as (38)], we could delete the other rows or columns of $\hat{\Omega}(a, b)$, (such as the second and fourth rows and columns for (38)). Then it would be sufficient to have $\varphi \geq 2$ for nonsingularity and for the pdf (39) to be well defined.

\section{EXAMPLE RESULTS}

To illustrate the theory derived above we shall consider both simulated and real data.

\section{A. Simulated Data}

For the simulated data we use a complex SOS autoregressive process $\left\{Z_{1, t}\right\}$ of order 1 , denoted CAR(1), with $\Delta=1$, and examine coherence of type (iii), i.e., that between $\left\{Z_{1, t}\right\}$ and $\left\{Z_{1, t}^{*}\right\}$. Simulation of the CAR(1) process is discussed in detail in [23]; the same parameter values were chosen, giving $\gamma_{*}^{2}(f)=$ 0.36 for all $|f|<1 / 2$. Since $R_{11}(f) \neq 0$ for all $|f|<1 / 2$, this CAR(1) process is an improper complex-valued SOS process [24]. As $\Delta=1$ here, $a=a_{0}$ and $b=b_{0}$ in what follows.
First, we consider analysis with a Morlet wavelet of the form (4) with $d=1$, a parameter choice which provides a "reasonable compromise between frequency and time resolution" [17, p. 3019]; see also [4, p. 139]. (The corresponding value of $f^{\prime}$ in (17) is 1.8.) The choice $\kappa_{0}=12$ gives approximately 10 degrees of freedom, (see Section IV-C), a sensible practical choice. To be more precise, every legitimate value $a_{\min } \leq a_{0} \leq a_{\max }$ will combine with the values of $d$ and $\kappa_{0}$ to produce a corresponding value of $N_{S}, N_{B}$ and $N_{P}$; see Table I. Combining these parameters with the Gaussian taper we obtain $\boldsymbol{B}$. As explained in Section IV-B, the complex degrees of freedom $\varphi$ can then be obtained from (34). These degrees of freedom are shown in Fig. 2(c); from a practical point of view it can be assumed that $\varphi=10$ for all $a_{0}$ values, as predicted in Section IV-C.

Realizations of the CAR(1) process used were of length $N=$ 1024. For each simulated sequence at each $(a, b)$ point

$$
\left[\begin{array}{cc}
\mathcal{S}\left\{Z_{1}, Z_{1}\right\} & \mathcal{S}\left\{Z_{1}, Z_{1}^{*}\right\} \\
\mathcal{S}\left\{Z_{1}^{*}, Z_{1}\right\} & \mathcal{S}\left\{Z_{1}^{*}, Z_{1}^{*}\right\}
\end{array}\right] \equiv\left[\begin{array}{cc}
\hat{S}_{11, b}(1 / a) & \hat{R}_{11, b}(1 / a) \\
\hat{R}_{11, b}^{*}(1 / a) & \hat{S}_{11, b}(-1 / a)
\end{array}\right]
$$

was calculated using (20). Then

$$
\hat{\gamma}_{*}^{2}(a, b)=\left|\hat{R}_{11, b}(1 / a)\right|^{2} /\left[\hat{S}_{11, b}(1 / a) \hat{S}_{11, b}(-1 / a)\right]
$$

was computed. This was repeated 100 times, and the ordered values of $\hat{\gamma}_{*}^{2}(a, b)$ obtained were plotted against the theoretical quantiles [14] of Goodman's distribution with $\gamma_{*}^{2}(a, b)=0.36$. Resulting quantile-quantile (Q-Q) plots are shown in Fig. 2(d) where the individual curves are for four different $(a, b)$ locations within the permitted ranges. At all locations $N_{P}>128$. The Q-Q plots indicate excellent agreement with Goodman's distribution.

For a second example, we use a different choice of $d$ and $\kappa_{0}$, but a choice which still gives approximately 10 degrees of freedom estimators. In line with the discussion in Section IV-C we can take $d=2$ and $\kappa_{0}=24$. The corresponding value of $f^{\prime}$ in (17) is 1.4. The complex degrees of freedom are shown in Fig. 3(c) and as expected $\varphi \approx 10$ for all $a_{0}$ values. For these parameters resulting Q-Q plots are shown in Fig. 3(d) and again indicate excellent agreement with Goodman's distribution.

\section{B. Solar Physics Data}

We consider two complex magnetic field time series, $\left\{Z_{1, n}\right\}$ and $\left\{Z_{2, n}\right\}$, recorded by two spacecraft in the solar physics 'Cluster' experiment. The component measured parallel to the ecliptic plane, and the component measured perpendicular, form the real and imaginary parts of the series of length about 1000 $\mathrm{s}$, with $\Delta=2 \mathrm{~s}$. For plots of the data see [16]. We examine coherence of type (i). The left plot of Fig. 5 shows the TWCOH estimate (36), while the right plot shows only values exceeding the $1 \%$ point of Goodman's distribution under the null hypothesis $\gamma^{2}(a, b)=0$, i.e., values $\hat{\gamma}^{2}(a, b)>1-\alpha^{1 /(\varphi-1)}$, for $\alpha=0.01, \varphi=10$. Clearly, there is nonnull coherence for scales exceeding 20. Since $f=1 / a$ here, this equates to coherent waves in the magnetic field for periods exceeding $20 \mathrm{~s}$.

\section{CONCLUding REMARKS}

Explicit expressions have been provided to show how in discrete-time the TWCOH estimator can be written in terms 

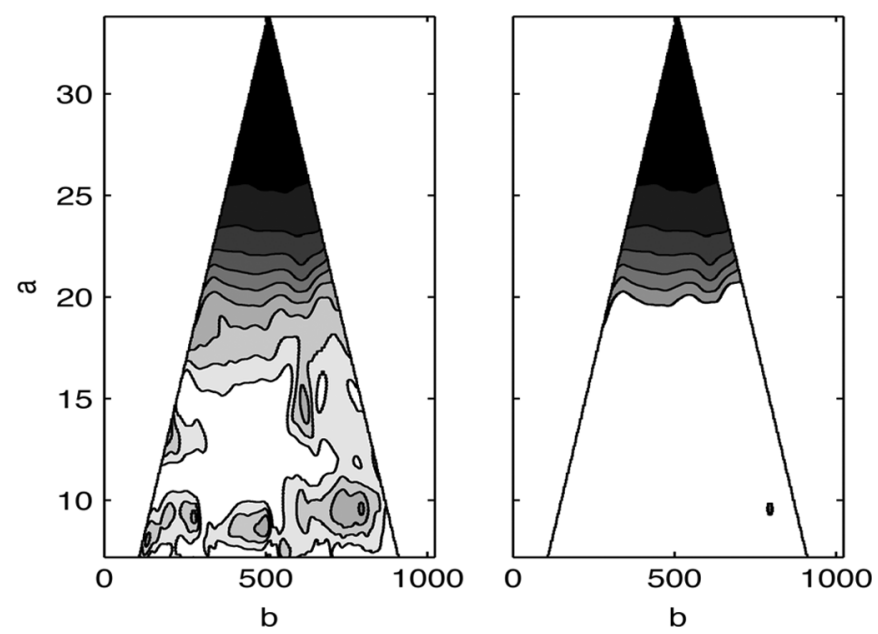

Fig. 5. TWCOH estimate of magnetic field data (left), and those values exceeding the $1 \%$ point of the null distribution (right). $d=1, \kappa_{0}=12, \varphi=10$. (Higher coherence corresponds to darker shading.)

of WOSA-type cross-spectral estimators, which can in turn be expressed in terms of multitaper estimators utilizing orthonormal tapers. Consequently, the number of complex degrees of freedom are immediately revealed. A fully specified Goodman's distribution results for the TWCOH estimator using a Morlet wavelet, applied to SOS complex-valued Gaussian processes.

While we have used a unit-norm Gaussian envelope to taper the complex exponential in the Morlet formulation, other sensible choices of taper function would fit into our general approach and the statistical analysis would proceed in the same way. In this sense the approach is quite general.

\section{APPENDIX}

\section{A. Proof of Theorem 1}

Proof: Write $Z_{l, n}=X_{l, n}+\mathrm{i} Y_{l, n}$, with $\left\{X_{l, n}\right\}$ and $\left\{Y_{l, n}\right\}$ real-valued, for $l=1,2$. Now define the real-valued vector $\left[X_{1, n}, X_{2, n}, Y_{1, n}, Y_{2, n}\right]^{T}=\left[\boldsymbol{X}_{n}^{T}, \boldsymbol{Y}_{n}^{T}\right]^{T}$. Then if

$$
\boldsymbol{T}=\left[\begin{array}{cc}
\boldsymbol{I}_{2} & \mathrm{i} \boldsymbol{I}_{2} \\
\boldsymbol{I}_{2} & -\mathrm{i} \boldsymbol{I}_{2}
\end{array}\right]
$$

we see that

$$
\boldsymbol{T}\left[\begin{array}{l}
\boldsymbol{X}_{n} \\
\boldsymbol{Y}_{n}
\end{array}\right]=\left[\begin{array}{l}
\boldsymbol{X}_{n}+\mathrm{i} \boldsymbol{Y}_{n} \\
\boldsymbol{X}_{n}-\mathrm{i} \boldsymbol{Y}_{n}
\end{array}\right]=\left[\begin{array}{c}
\boldsymbol{Z}_{n} \\
\boldsymbol{Z}_{n}^{*}
\end{array}\right]=\check{\boldsymbol{Z}}_{n}
$$

Form the product $\left\{u_{j, n} \check{Z}_{n},\right\}$ of the $j$ th real-valued taper with $\check{Z}_{n}$, and then compute its (scaled) Fourier transform

$$
\check{\boldsymbol{J}}_{j}(f)=\Delta^{1 / 2} \sum_{n=0}^{N-1} u_{j, n}\left[\begin{array}{c}
\boldsymbol{Z}_{n} \\
\boldsymbol{Z}_{n}^{*}
\end{array}\right] \mathrm{e}^{-\mathrm{i} 2 \pi f n \Delta}=\left[\begin{array}{c}
\boldsymbol{J}_{j}(f) \\
\boldsymbol{J}_{j}^{*}(-f)
\end{array}\right]
$$

where $\boldsymbol{J}_{j}(f) \equiv \Delta^{1 / 2} \sum_{n=0}^{N-1} u_{j, n} \boldsymbol{Z}_{n} \mathrm{e}^{-\mathrm{i} 2 \pi f n \Delta}$. From (41)

$$
\check{\boldsymbol{J}}_{j}(f)=\boldsymbol{T} \Delta^{1 / 2} \sum_{n=0}^{N-1} u_{j, n}\left[\begin{array}{l}
\boldsymbol{X}_{n} \\
\boldsymbol{Y}_{n}
\end{array}\right] \mathrm{e}^{-\mathrm{i} 2 \pi f n \Delta}=\boldsymbol{T}\left[\begin{array}{c}
\boldsymbol{J}_{X, j}(f) \\
\boldsymbol{J}_{Y, j}(f)
\end{array}\right]
$$

where $\boldsymbol{J}_{X, k}(f) \equiv \Delta^{1 / 2} \sum_{n=0}^{N-1} u_{j, n} \boldsymbol{X}_{n} \mathrm{e}^{-\mathrm{i} 2 \pi f n \Delta}$ and similarly for $\boldsymbol{J}_{Y, j}(f)$. Hence

$$
\left[\begin{array}{c}
\boldsymbol{J}_{j}(f) \\
\boldsymbol{J}_{j}^{*}(-f)
\end{array}\right]=\boldsymbol{T}\left[\begin{array}{l}
\boldsymbol{J}_{X, j}(f) \\
\boldsymbol{J}_{Y, j}(f)
\end{array}\right]
$$

and as an estimator of $\boldsymbol{\Upsilon}(f)$ we take the multitaper multivariate estimator $\hat{\boldsymbol{\Upsilon}}(f)=\left(1 / N_{B}\right) \sum_{j=0}^{N_{B}-1} \gamma_{j} \check{\boldsymbol{J}}_{j}(f) \check{\boldsymbol{J}}_{j}^{H}(f)$, which can be written as

$$
\frac{1}{N_{B}} \sum_{j=0}^{N_{B}-1} \gamma_{j}\left[\begin{array}{cc}
\boldsymbol{J}_{j}(f) \boldsymbol{J}_{j}^{H}(f) & \boldsymbol{J}_{j}(f) \boldsymbol{J}_{j}^{T}(-f) \\
\left\{\boldsymbol{J}_{j}(-f) \boldsymbol{J}_{j}^{T}(f)\right\}^{*} & \left\{\boldsymbol{J}_{j}(-f) \boldsymbol{J}_{j}^{H}(-f)\right\}^{*}
\end{array}\right]
$$

and (42) means that also $\hat{\Upsilon}(f)$ can also be written as

$$
\frac{1}{N_{B}} \sum_{j=0}^{N_{B}-1} \gamma_{j} \boldsymbol{T}\left[\begin{array}{ll}
\boldsymbol{J}_{X, j}(f) \boldsymbol{J}_{X, j}^{H}(f) & \boldsymbol{J}_{X, j}(f) \boldsymbol{J}_{Y, j}^{H}(f) \\
\boldsymbol{J}_{Y, j}(f) \boldsymbol{J}_{X, j}^{H}(f) & \boldsymbol{J}_{Y, j}(f) \boldsymbol{J}_{Y, j}^{H}(f)
\end{array}\right] \boldsymbol{T}^{H}
$$

so that

$$
\hat{\boldsymbol{\Upsilon}}(f)=\left[\begin{array}{cc}
\hat{\boldsymbol{S}}(f) & \hat{\boldsymbol{R}}(f) \\
\hat{\boldsymbol{R}}^{H}(f) & \hat{\boldsymbol{S}}^{T}(-f)
\end{array}\right]=\boldsymbol{T} \hat{\boldsymbol{\Sigma}}(f) \boldsymbol{T}^{H}
$$

where

$$
\hat{\boldsymbol{\Sigma}}(f) \equiv\left[\begin{array}{ll}
\hat{\boldsymbol{S}}_{X, X}(f) & \hat{\boldsymbol{S}}_{X, Y}(f) \\
\hat{\boldsymbol{S}}_{Y, X}(f) & \hat{\boldsymbol{S}}_{Y, Y}(f)
\end{array}\right]
$$

and, e.g., $\hat{\boldsymbol{S}}_{X, Y}(f) \equiv\left(1 / N_{B}\right) \sum_{j=0}^{N_{B}-1} \gamma_{j} \boldsymbol{J}_{X, j}(f) \boldsymbol{J}_{Y, j}^{H}(f)$. Since $\left[\boldsymbol{X}_{n}^{T}, \boldsymbol{Y}_{n}^{T}\right]^{T}$ is real-valued and Gaussian, it follows immediately [27, p. 776] that asymptotically we may take the distribution of $\hat{\boldsymbol{\Sigma}}(f)$ to be

$$
\hat{\boldsymbol{\Sigma}}(f) \stackrel{\mathrm{d}}{=}(1 / \varphi) \mathcal{W}_{4}^{C}\{\varphi, \boldsymbol{\Sigma}(f)\}, \quad 0<|f|<f_{\mathcal{N}} .
$$

Here $\mathcal{W}_{4}^{C}\{\varphi, \boldsymbol{\Sigma}(f)\}$ denotes the four-dimensional complex central Wishart distribution with $\varphi$ complex degrees of freedom and mean $\varphi \boldsymbol{\Sigma}(f) . \boldsymbol{\Sigma}(f)$ is the true spectral matrix for the process $\left\{\left[\boldsymbol{X}_{n}^{T}, \boldsymbol{Y}_{n}^{T}\right]^{T}\right\}$.

We want the distribution of $\hat{\boldsymbol{\Upsilon}}(f)=\boldsymbol{T} \hat{\boldsymbol{\Sigma}}(f) \boldsymbol{T}^{H}$. Now $\boldsymbol{\Upsilon}(f)=\boldsymbol{T} \boldsymbol{\Sigma}(f) \boldsymbol{T}^{H}$. Combining this with (44) and the result of Lemma 1 given below we see that asymptotically

$$
\hat{\boldsymbol{\Upsilon}}(f) \stackrel{\mathrm{d}}{=}(1 / \varphi) \mathcal{W}_{4}^{C}\{\varphi, \Upsilon(f)\}, \quad 0<|f|<f_{\mathcal{N}}
$$

Lemma 1: If $\boldsymbol{W}$ has the $\mathcal{W}_{q}^{C}\{\ell, \boldsymbol{\Sigma}\}$ distribution and $\boldsymbol{M}$ is a complex-valued $q \times q$ matrix, then $\boldsymbol{M} \boldsymbol{W} \boldsymbol{M}^{H}$ has the $\mathcal{W}_{q}^{C}\left\{\ell, \boldsymbol{M} \boldsymbol{\Sigma} \boldsymbol{M}^{H}\right\}$ distribution.

Proof: The characteristic function of $W$ is $([9$, p. 163) ]

$$
\begin{aligned}
\phi\left(\boldsymbol{\Theta}_{0}\right) & =E\left\{\exp \left[\mathrm{i} \cdot \operatorname{tr}\left(\boldsymbol{W} \boldsymbol{\Theta}_{0}\right)\right]\right\} \\
& =\left[\operatorname{det}\left(\boldsymbol{I}_{q}-\mathrm{i} \boldsymbol{\Theta}_{0} \boldsymbol{\Sigma}\right)\right]^{-\ell}
\end{aligned}
$$

The characteristic function, $\phi(\Theta)$, of $\boldsymbol{M} \boldsymbol{W} \boldsymbol{M}^{H}$ is therefore

$$
\begin{aligned}
E\left\{\exp \left[\mathrm{i} \cdot \operatorname{tr}\left(\boldsymbol{M} \boldsymbol{W} \boldsymbol{M}^{H} \boldsymbol{\Theta}\right)\right]\right\} & =E\left\{\exp \left[\mathrm{i} \cdot \operatorname{tr}\left(\boldsymbol{W} \boldsymbol{M}^{H} \boldsymbol{\Theta} \boldsymbol{M}\right)\right]\right\} \\
& =\left[\operatorname{det}\left(\boldsymbol{I}_{q}-\mathrm{i} \boldsymbol{M}^{H} \boldsymbol{\Theta} \boldsymbol{M} \boldsymbol{\Sigma}\right)\right]^{-\ell} \\
& =\left[\operatorname{det}\left(\boldsymbol{I}_{q}-\mathrm{i} \boldsymbol{\Theta} \boldsymbol{M} \boldsymbol{\Sigma} \boldsymbol{M}^{H}\right)\right]^{-\ell}
\end{aligned}
$$


where tr and det denote trace and determinant, respectively, and we have used the fact that if $\boldsymbol{A}$ is an $j \times k$ complex-valued matrix and $B$ is an $k \times j$ complex-valued matrix, then $\operatorname{tr}(\boldsymbol{A} \boldsymbol{B})=\operatorname{tr}(\boldsymbol{B} \boldsymbol{A})$ and $\operatorname{det}\left(\boldsymbol{I}_{j}+\boldsymbol{A} \boldsymbol{B}\right)=\operatorname{det}\left(\boldsymbol{I}_{k}+\boldsymbol{B} \boldsymbol{A}\right)$, [7]. Comparing (46) and (47), it follows that $\boldsymbol{M W} \boldsymbol{M}^{H}$ has the $\mathcal{W}_{q}^{C}\left\{\ell, M \Sigma M^{H}\right\}$ distribution.

\section{ACKNOWLEDGMENT}

The authors thank the referees and Associate Editor for their suggestions and comments.

\section{REFERENCES}

[1] R. Bortel and P. Sovka, "Approximation of statistical distribution of magnitude squared coherence estimated with segment overlapping," Signal Process., vol. 87, pp. 1100-1117, 2007.

[2] J.-S. Brittain, D. M. Halliday, B. A. Conway, and J. B. Nielsen, "Singletrial multiwavelet coherence in application to neurophysiological time series," IEEE Trans. Biomed. Eng., vol. 54, pp. 854-862, 2007.

[3] T. P. Bronez, "On the performance advantage of multitaper spectral analysis," IEEE Trans. Signal Process., vol. 40, pp. 2941-2946, 1992.

[4] R. Carmona, W.-L. Hwang, and B. Torrésani, Practical Time-Frequency Analysis. San Diego, CA: Academic, 1998.

[5] G. C. Carter, "Coherence and time delay estimation," Proc. IEEE, vol. 75, pp. 236-255, 1987.

[6] E. A. K. Cohen and A. T. Walden, "A statistical analysis of Morse wavelet coherence," IEEE Trans. Signal Process., vol. 58, pp. 980-989, 2010.

[7] M. L. Eaton, Multivariate Statistics. New York: Wiley, 1983.

[8] W. Gao and B. Li, "Wavelet analysis of coherent structures at the atmosphere-forest interface," J. Appl. Meteorol., vol. 32, pp. 1717-1725, 1993.

[9] N. R. Goodman, "Statistical analysis based on a certain multivariate complex Gaussian distribution (an introduction)," Ann. Math. Statist., vol. 34, pp. 152-177, 1963.

[10] I. S. Gradshteyn and I. M. Ryzhik, Table of Integrals, Series, and Products (Corrected and Enlarged Edition). New York: Academic, 1980.

[11] A. Grinsted, J. C. Moore, and S. Jevrejeva, "Application of the cross wavelet transform and wavelet coherence to geophysical time series," Nonlin. Processes in Geophys., vol. 11, pp. 561-566, 2004.

[12] K. Gurley, T. Kijewski, and A. Kareem, "First- and higher-order correlation detection using wavelet transforms," ASCE J. Eng. Mechan., vol. 129, pp. 188-201, 2003.

[13] J.-Ph. Lachaux, A. Lutz, D. Rudrauf, D. Cosmelli, M. Le Van Quyen, J. Martinerie, and F. Varela, "Estimating the time-course of coherence between single-trial brain signals: An introduction to wavelet coherence," Clin. Neurophys., vol. 32, pp. 157-174, 2002.

[14] P. F. Lee, "An algorithm for computing the cumulative distribution function for magnitude squared coherence estimation," IEEE Trans. Acoust., Speech Signal Process., vol. 29, pp. 117-119, 1981.

[15] P. C. Liu, "Wavelet spectrum analysis and ocean wind waves," in Wavelets in Geophysics, E. Foufoula-Georgiou and P. Kumar, Eds. San Diego, CA: Academic, 1994.
[16] T. Medkour and A. T. Walden, "A variance equality test for two correlated complex Gaussian variables with applications to spectral power comparisons," IEEE Trans. Signal Process., vol. 55, pp. 881-888, 2007.

[17] B.-Ph. van Milligen, E. Sánchez, T. Estrada, C. Hidalgo, B. Brañas, B. Carreras, and L. García, "Wavelet bicoherence: A new turbulence analysis tool," Phys. Plasmas, vol. 2, pp. 3017-3032, 1995.

[18] E. W. Montroll and M. F. Shlesinger, "Maximum entropy formalism, fractals, scaling phenomena, and 1 / $f$ noise: A tale of tails," J. Statist. Phys., vol. 32, pp. 3380-3383, 1983.

[19] C. N. K. Mooers, "A technique for the cross spectrum analysis of pairs of complex-valued time series, with emphasis on properties of polarized components and rotational invariants," Deep-Sea Res., vol. 20, pp. 1129-1141, 1973.

[20] A. H. Nuttall and G. C. Carter, "Spectral estimation using combined time and lag weighting," Proc. IEEE, vol. 70, pp. 1115-1125, 1982.

[21] S. C. Olhede and A. T. Walden, "Noise reduction in directional signals using multiple Morse wavelets illustrated on quadrature Doppler ultrasound," IEEE Trans. Biomed. Eng., vol. 50, pp. 51-57, 2003.

[22] M. I. Plett, "Transient detection with cross wavelet transforms and wavelet coherence," IEEE Trans. Signal Process., vol. 55, pp. 1605-1611, 2007.

[23] P. Rubin-Delanchy and A. T. Walden, "Simulation of improper complex-valued sequences," IEEE Trans. Signal Process., vol. 55, pp. 5517-5521, 2007.

[24] P. J. Schreier and L. L. Scharf, "Second-order analysis of improper complex random vectors and processes," IEEE Trans. Signal Process., vol. 51, pp. 714-725, 2003.

[25] G. Strang, Linear Algebra and its Applications, 3rd ed. Orlando, FL: Harcourt Brace Jovanovich.

[26] C. Torrence and P. Webster, "Interdecadal changes in the ENSO-Monsoon system," J. Climate, vol. 12, pp. 2679-2690, 1999.

[27] A. T. Walden, "A unified view of multitaper multivariate spectral estimation," Biometrika, vol. 87, pp. 767-787, 2000.

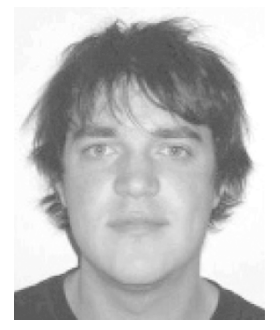

E. A. K. Cohen received the M.Phys. degree in mathematics and physics from the University of Warwick, Coventry, U.K., in 2005.

He worked as a Signal Processing Scientist with QinetiQ, Malvern, U.K., for two years before beginning his Ph.D. studies at Imperial College London, U.K., in 2007. His interests include multichannel signals and wavelet applications in signal processing.

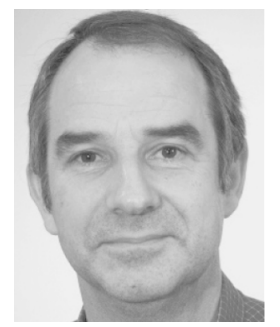

A. T. Walden (A'86-M'07) received the B.Sc degree in mathematics from the University of Wales, Bangor, U.K., in 1977, and the M.Sc. and $\mathrm{Ph} . \mathrm{D}$. degrees in statistics from the University of Southampton, Southampton, U.K., in 1979 and 1982, respectively.

He was a Research Scientist at BP, London, U.K., from 1981 to 1990 . He then joined the Department of Mathematics, Imperial College London, London, where he is currently a Professor of statistics. 